\title{
Representation of time in time-place learning
}

\author{
MATTHEW J. PIZZO and JONATHON D. CRYSTAL \\ University of Georgia, Athens, Georgia
}

\begin{abstract}
Ordinal, interval, and circadian mechanisms of solving a time-place task were tested. Rats searched for food twice in the morning and once in the afternoon (Group $\mathrm{AB}-\mathrm{C}, n=5$ ) or once in the morning and twice in the afternoon (Group A-BC, $n=5$ ) in a box with four food troughs. The location of the food depended on the time of day in a 12:12-h light:dark cycle. Acquisition was documented by foodsite inspections at the correct locations prior to food availability. On nonrewarded probes, the time of the middle search (B) was shifted late (for Group AB-C) or early (for Group A-BC). The rats visited Location $\mathrm{B}$ at chance, contrary to an ordinal mechanism. When the posttesting meal and light-dark transitions were omitted, the rats visited correct locations with impaired performance but at abovechance levels on nonrewarded probes. The results are consistent with interval and circadian representations of time.
\end{abstract}

A fundamental question about time perception concerns the form in which time is represented. Psychophysical approaches to answering this and related questions have focused on the various scales of representation that might be available to an animal. For example, Stevens (1951) described a hierarchy of measurement scales ranging from nominal to ordinal, interval, and ratio. In this hierarchy, higher order scales contain the properties of lower order scales plus the addition of an extra property. For example, with an ordinal scale, it is possible to represent the order of values on the scale. An interval scale has added to it information about the spacing of successive values, permitting the addition and subtraction of values on the scale. Finally, a ratio scale has added to it information about the relative position of values on a scale with an absolute zero point, permitting the multiplication and division of values on the scale. For example, finalists in a race are ranked first, second, and third. This ordinal ranking conveys no information about how close the finish times were among the finalists. Therefore, there is no guarantee of a meaningful outcome if higher order operations are performed on these ranks. However, in some cases, a meaningful outcome might occur (e.g., when the finish times for the finalists are about equally spaced).

Identifying the representation of time is an important issue for theories of timing. For example, in the scalar

The data were collected in partial fulfillment of the MS degree by M.J.P. and were presented at the 42nd Annual Meeting of the Psychonomic Society, Orlando, Florida, November 2001. This work was supported by National Institutes of Mental Health Grant MH61618 to J.D.C. The raw data (time of occurrence of each stimulus and response) are available at www.uga.edu/animal-cognition-lab. We thank Kenneth W. Maxwell for assistance with data analysis. We thank Peter Killeen for helpful comments on the manuscript. Correspondence should be addressed to M. J. Pizzo or J. D. Crystal, Department of Psychology, University of Georgia, Athens, GA 30602-3013 (e-mail: mpizzo@arches. uga.edu; jcrystal@uga.edu). timing theory (Gibbon, 1991), it is proposed that an accumulator integrates the number of pulses from a pacemaker. According to the behavioral theory of timing (Killeen \& Fetterman, 1988), an animal proceeds through a sequence of behavioral states with transitions produced by a pacemaker. Staddon and Higa (1999) proposed that timing abilities are based on the magnitude of a memory trace since the last reinforcement. Theories of timing have generally not explicitly stated what type of measurement scale should be used. In some cases, however, a theory can be more compatible with one measurement scale than with others. For example, an important feature of scalar timing theory is the use of ratio decision rules, which are naturally compatible with a ratio scale. However, in other cases, the compatibility is less clear. For example, although the behavioral theory of timing is based on an ordinal sequence of behaviors, the theory has been used successfully in a variety of tasks that imply higher order scales (e.g., it can predict bisection at the geometric mean, which implies a ratio scale).

One approach to identifying the representation of time involves a psychophysical method. For example, in Gibbon and Church's (1981) time-left procedure, animals were required to choose between a standard interval (e.g., a fixed $30 \mathrm{sec}$ ) and a comparison interval (e.g., $60-t$, where $t$ is the elapsed time into the trial when the comparison lever is inserted). For example, in one of their experiments, a trial began with the comparison lever entering the chamber followed by the standard lever entering at 15,30 , or $45 \mathrm{sec}$ into the interval. The rats chose the standard lever when it entered early, at $15 \mathrm{sec}$, and the comparison lever when the standard entered at $45 \mathrm{sec}$. When the standard and comparison intervals were varied across conditions, the point of subjective equality (i.e., equal likelihood of choosing standard and comparison) changed with standard and comparison intervals. Gibbon and Church argued that these results imply that time is measured on a linear rather than on a logarithmic scale. 
Although this argument has recently been criticized (see Staddon \& Higa, 1999), it has also been extended to numerical discrimination in pigeons (Brannon, Wusthoff, Gallistel, \& Gibbon, 2001) and to timing in humans (Wearden, 2002).

Another approach to investigating the representation of time involves the evaluation of timing in a foraging context. For example, in a time-place learning task, food availability depends on temporal and spatial information. Time-place learning has been observed in a variety of species (see Biebach, Gordijn, \& Krebs, 1989; Carr \& Wilkie, 1997b; Mistlberger, de Groot, Bossert, \& Marchant, 1996; Saksida \& Wilkie, 1994; Widman, Gordon, \& Timberlake, 2000; Wilkie \& Willson, 1992). For example, Biebach et al. (1989) tested garden warblers in a central room connected to four adjacent rooms. Food was available in each of the rooms at four different times of day. The birds primarily restricted their visits to the room that currently provided food, but the birds also demonstrated anticipation of food availability by visiting each room before the food became available at that location. Performance reached a criterion of $75 \%$, suggesting that the birds learned the association of each room with its rewarded time of day. In a subsequent experiment, food was available at each of the four rooms continuously throughout the day. The purpose of this test was to assess whether the birds were using periodic food availability as transition cues. The test revealed a preference for each room at its correct time of day.

In another study, Krebs and Biebach (1989) focused on whether birds used a time-place mechanism, and they tested the hypothesis that the birds were relying on a fixed route by blocking some of the locations where food was available. Their testing this alternative explanation is particularly important because animals are capable of learning serial patterns (see Church \& Lacourse, 1998; Fountain, Benson, \& Wallace, 2000). Access to all four rooms was restricted from $0600 \mathrm{~h}$ to $1000 \mathrm{~h}$ and from $1200 \mathrm{~h}$ to $1600 \mathrm{~h}$, but this did not prevent the birds from visiting the correct locations at the other times of the day when access to the rooms was available.

Biebach, Falk, and Krebs (1991) focused on whether animals time with an interval or a circadian clock and investigated whether changes in light affect performance. Constant dim light and phase-delay manipulations suggested that the subjects (garden warblers) used a circadian oscillator to solve the time-place task. Mistlberger et al. (1996) also found evidence to support a circadian mechanism in a time-place task with rats. Reinforcement was given when the rats selected one end of a T-maze in the morning and the opposite end in the afternoon. The rats attained a high level of performance that was not disrupted by inverting the light:dark cycle or by omitting 1-3 daily sessions. This result suggests that the order in which the events occurred, the light transitions, or an alternation strategy was not used to solve this task.

Recently, Carr and Wilkie (1997a, 1997b; see also Carr, Tan, \& Wilkie, 1999) argued that rats' performance in time-place tasks is in part based on an ordinal representation of time. They trained rats to press a lever at two locations within a chamber. The rats learned to go to the first location at $0930 \mathrm{~h}$ and to the second location at $1530 \mathrm{~h}$. After a $0930 \mathrm{~h}$ session was omitted, they found that at $1530 \mathrm{~h}$ the rats incorrectly leverpressed at the $0930 \mathrm{~h}$ location. When a $1530 \mathrm{~h}$ session was skipped, and testing occurred at $0930 \mathrm{~h}$, the rats leverpressed at the $0930 \mathrm{~h}$ location. Carr and Wilkie (1997a, 1997b) argued that these results imply an ordinal representation of time, because in both cases, the animals started daily testing by going to the first location of the day. According to this proposal, the rat resets its ordinal timer each day by consulting a circadian oscillator and visits specific locations by consulting an ordinal timer.

\section{EXPERIMENT 1}

The present study was designed to test a prediction of an ordinal representation of time. If an animal uses an ordinal scale of measurement, it should be insensitive to transformations that require a higher order scale. For example, there is no guarantee that addition or subtraction on an ordinal scale will produce meaningful results. Therefore, a rat's using an ordinal scale should be insensitive to an additive transformation of time.

In the present study, the rats searched for food at three fixed times per day. The location of food depended on the time of the shift. For example, for a given animal, food was available at Locations 1, 2, and 3 in Shifts A, $\mathrm{B}$, and C, respectively. At a fourth location, food was never provided. The time of testing the middle shift was unusually early or late on occasional nonrewarded probes. If an animal was solving the time-place task by tracking the temporal order of spatial locations, an early or late test should not interfere with performance: The animal should search at the second location as it usually does on the second test of the day. In contrast, if an animal was using an interval or circadian mechanism, testing at a new time should produce a decline to chance performance. An intermediate result might suggest the operation of multiple timing mechanisms.

\section{Method}

Subjects. Ten male Long-Evans rats were obtained from Harlan (Madison, WI). The rats were experimentally naive and 93 days old at the beginning of the experiment. The rats were given 5001 Rodent Diet (Lab Diet, Brentwood, MO) ad lib for 1 week, followed by $20 \mathrm{~g}$ for 1 day, and $15 \mathrm{~g}$ per day thereafter. They were given free access to water, except during brief experimental sessions. The average weight was $284 \mathrm{~g}$ at the beginning of the experiment. The rats were individually housed in clear plastic cages lined with bedding (Andersons, Bed o'Cobs, Maumee, $\mathrm{OH}$ ). The colony was subjected to a 12:12-h light:dark cycle with light onset and offset at $0700 \mathrm{~h}$ and $1900 \mathrm{~h}$, respectively.

Apparatus. An open-field box $(43.2 \times 43.2 \mathrm{~cm}$ PVC base $)$ with clear acrylic walls (30.5 cm high, MED Associates, ENV-515, Georgia, VT) was covered with clear acrylic. A food trough (MED Associates, ENV-200R2M) was positioned in the center of each wall, $7 \mathrm{~cm}$ above the floor, which consisted of a $1.27 \times 1.27 \mathrm{~cm}$ 
metal grid. A photobeam (MED Associates, ENV-254), situated $1 \mathrm{~cm}$ inside the trough $(1 \mathrm{~cm}$ from the bottom of the trough $) \mathrm{de}-$ tected head entries into each food trough. A response was recorded at the time that the photobeam was first interrupted; another response could not be recorded until the interruption had terminated. A food dispenser (MED Associates, ENV-203IR) was connected to each food trough outside of the box. The open field was placed on a table $(74 \mathrm{~cm}$ above the floor) centered in a $3.7 \times 4.0 \mathrm{~m}$ room. There were several objects in the room (i.e., desk, chair, sink, shelves) that remained in fixed locations throughout the experiment. In an adjacent room, a Celeron 500-MHz computer running MED-PC for Windows (Version 1.15) controlled the experimental events and recorded the events with 10 -msec resolution.

Procedure. The rats were individually placed in the box three times per day (Shifts A, B, and C), at approximately the same times each day. For each rat, three locations were active and one was inactive. Food (45-mg pellets, Improved Formula A/I; P. J. Noyes Co., Lancaster, $\mathrm{NH}$ ) was available at one active location for each shift. The active locations were randomly assigned to shift times for each rat prior to the start of the experiment. The rats were randomly assigned to groups, with the constraint that each group was to have 5 rats. All rats were tested in the morning and the afternoon (Shifts A and $C$, respectively). The time at which Shift B occurred varied across groups. In Group AB-C, Shift B occurred immediately after Shift A (i.e., in the morning). In Group A-BC, Shift B occurred immediately before Shift $\mathrm{C}$ (i.e., in the afternoon). The apparatus was cleaned once per day.

Daily testing began at $0900 \mathrm{~h}$. Group A-BC's Shift A occurred first. This was immediately followed by Group AB-C's Shifts A and B. Testing resumed in the afternoon, at approximately $1520 \mathrm{~h}$, at which time Group A-BC's Shifts B asnd C were tested. Finally, Group AB-C's Shift C was tested. This testing arrangement equated short and long intervals between the shifts for each group. The mean short and long intervals were $0.55 \mathrm{~h}(S E=0.02)$ and $6.21 \mathrm{~h}(S E=0.03)$, respectively.

On the initial day of training, 10 pellets were dispensed at the scheduled active location with a 60 -sec interfood interval during each shift. On this day only, a head-entry response had no effect on the delivery of food. On the 2 nd day of training, the rats received a 1-sec fixed interval (FI) schedule: The first reward was delivered after the first response (i.e., head entry) after $1 \mathrm{sec}$ at the active location. The session ended when 25 reinforcements were obtained or when $10 \mathrm{~min}$ had elapsed. In the third session, the FI was replaced by two fixed ratio (FR) schedules. The first pellet was earned on an FR1 schedule; the remaining pellets in the session were earned on an FR5 schedule. The shift ended after 26 reinforcements or when $10 \mathrm{~min}$ had elapsed. An important factor in the development of time-place discrimination is the effect of response cost. Widman et al. (2000) found that high response costs promoted time-place discrimination. Therefore, schedules were gradually increased until each rat earned the first and subsequent pellets on an FR70 and an FR10 schedule, respectively. All rats reached these criteria within 42 days. The data consist of the number of photobeam interruptions at each location during the initial FR (i.e., prior to the first pellet). After each shift, the rat was returned to the colony, and the next rat was tested. The rats received $15 \mathrm{~g}$ of food at the end of the day, approximately 20-30 min after Shift C. This meal occurred at approximately $1700 \mathrm{~h}$. The daily testing was conducted at a fixed time and fixed sequence each day, 6-7 days per week. Acquisition took 76 sessions.

On occasional nonrewarded probes, Shift B for Group AB-C was conducted unusually late in the day; Shift B for Group A-BC was conducted unusually early in the day. During these probe shifts, responses were recorded until any single location registered 70 responses, and pellets were not delivered; therefore, probe shifts were identical to the initial portion of training shifts (i.e., both types of shift had a location with 70 nonreinforced responses). The rats were tested during Shifts $\mathrm{A}$ and $\mathrm{C}$ as in training. Three probe shifts were conducted. Intermixed with these probe days were training days that were identical to those of acquisition described above. Each probe was randomized within a 7-day block with the restriction that probes were separated by at least 3 consecutive training days.

Throughout this article, the criterion for statistical significance is .05 .

\section{Results}

In Figure 1, the proportion of photobeam breaks that occurred at the correct location (photobeam breaks at the correct location/sum of photobeam breaks at all locations) are plotted as a function of five-session blocks. Performance was initially near the chance level of .25 (chance was defined as .25 because there were four food troughs in the box). By the end of training, performance was $.894(S E=.021)$ and $.943(S E=.028)$ for Groups $\mathrm{AB}-\mathrm{C}$ and $\mathrm{A}-\mathrm{BC}$, respectively.

An analysis of variance (ANOVA) with session and shift as the repeated measures and group as the betweensubjects measure revealed that there was a significant effect of session $[F(14,112)=47.45]$ and of shift $[F(2,16)=7.48]$. The main effect of group was not significant, nor were the interactions. Paired $t$ tests were used to characterize the effect of shift. Performance during Shifts B and C was reliably greater $[t(9)=2.73$, and $t(9)=3.99$, respectively] than during Shift A. There was no significant difference between Shifts B and C.

In Figure 2, the data are plotted from training and nonrewarded probe-B shifts. The baseline data consist of the proportion of correct responses in Shift B in the last fivesession block prior to probe testing. These data were submitted to a $2 \times 2$ ANOVA with condition (probe vs. baseline) as the repeated measure and groups as the between measure. There was a significant effect of condition $[F(1,8)=42.14]$. There was no significant effect of group and no group $\times$ condition interaction. The mean difference between baseline and probe was .675 ( $S E=$ .094). Inspection of the B location was significantly lower on probe tests relative to baseline $[t(9)=7.15]$. On probe tests, food-trough inspections at the $\mathrm{B}$ location did not differ from the chance level of .25 . The same conclusions were reached when the analysis was restricted to the initial $60 \mathrm{sec}$ of data.

\section{Discussion}

Testing at an unusually early or late time produced a decline in performance to chance levels. The rats were sensitive to the temporal placement of the middle shift, contrary to the prediction of an ordinal representation of time. This sensitivity could be based on the rats' measuring the interval between Shifts A and B or to the time of day of B. According to an ordinal representation, the rats were expected to search at the B location when the second shift occurred. The decline in performance on probe tests is consistent with the use of an interval or circadian mechanism. According to a circadian mechanism, the phase at which Shift B occurred in the rats' circadian cycle was changed during the probe tests. According to 


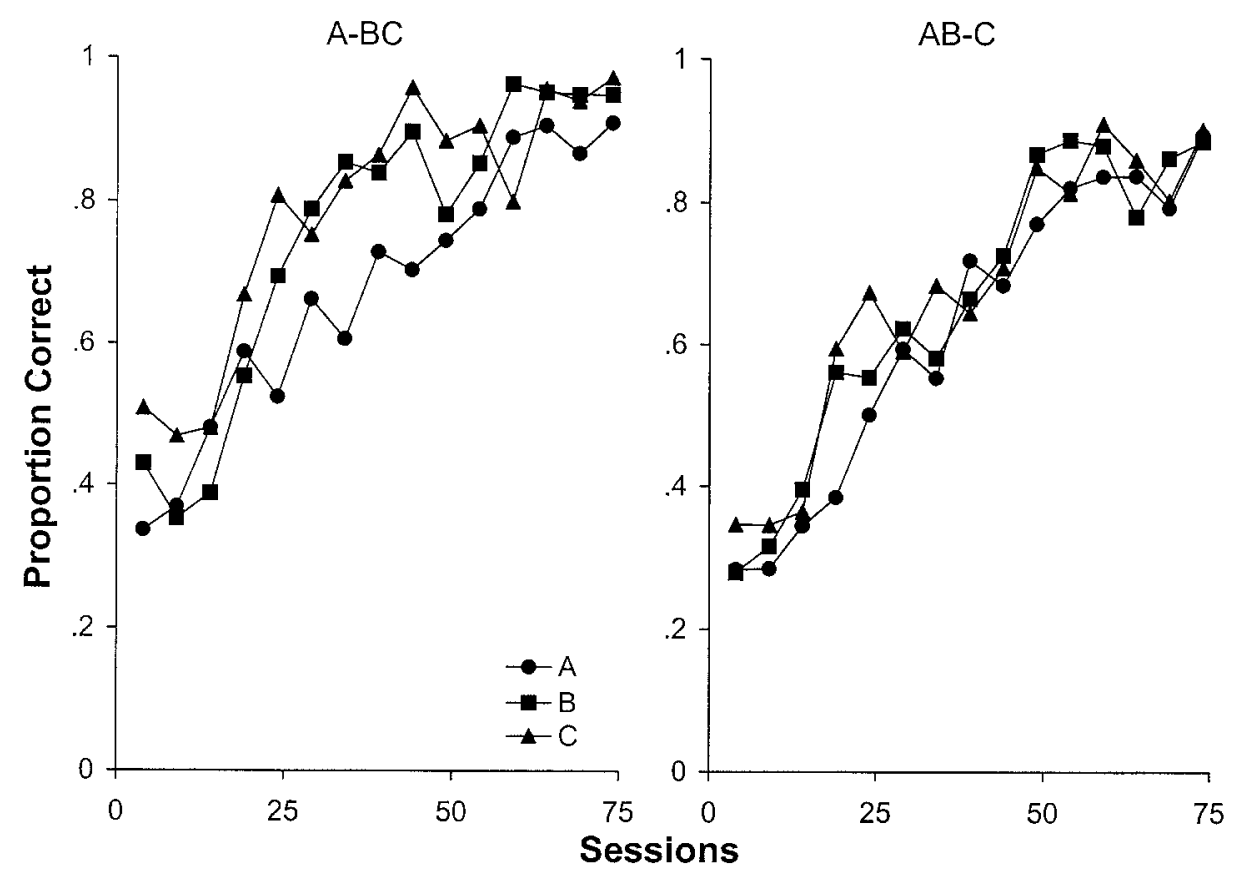

Figure 1. Mean proportion of photobeam breaks at the correct location for each shift during acquisition plotted as a function of blocks of five sessions in Experiment 1.

an interval mechanism, the interval at which Shift B occurred was changed during the probe tests.

\section{EXPERIMENT 2}

In Experiment 1, it was demonstrated that the rats were not timing with respect to an ordinal representation of time, yet it is not clear whether the rats were using an internal or a circadian mechanism. The rats might solve the time-place task by timing the interval with respect to the presentation of a stimulus. Potential salient stimuli include light onset in the colony, the postsession daily ration of food from the previous day, and food at the active locations on the current day. Alternatively, these cues could be timed by

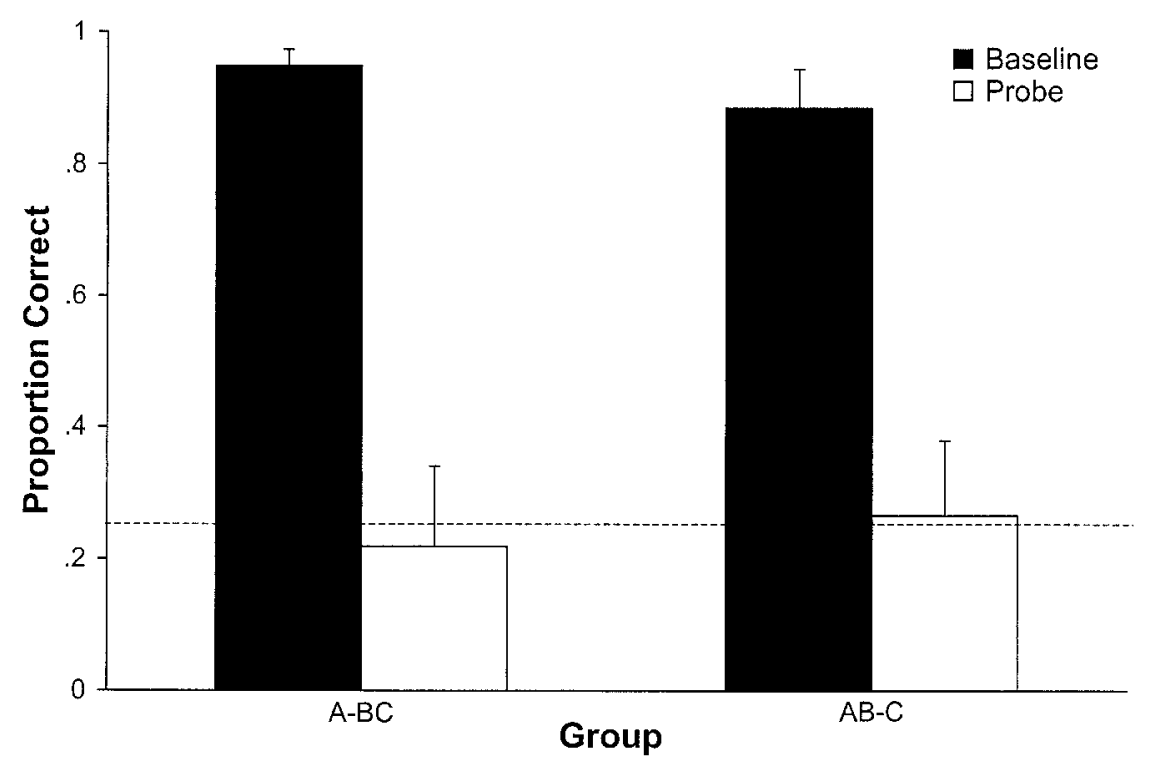

Figure 2. Mean proportion of responses at the $B$ location during the second shift of the day in Experiment 1. The dashed line represents chance performance; error bars represent $S E$. 


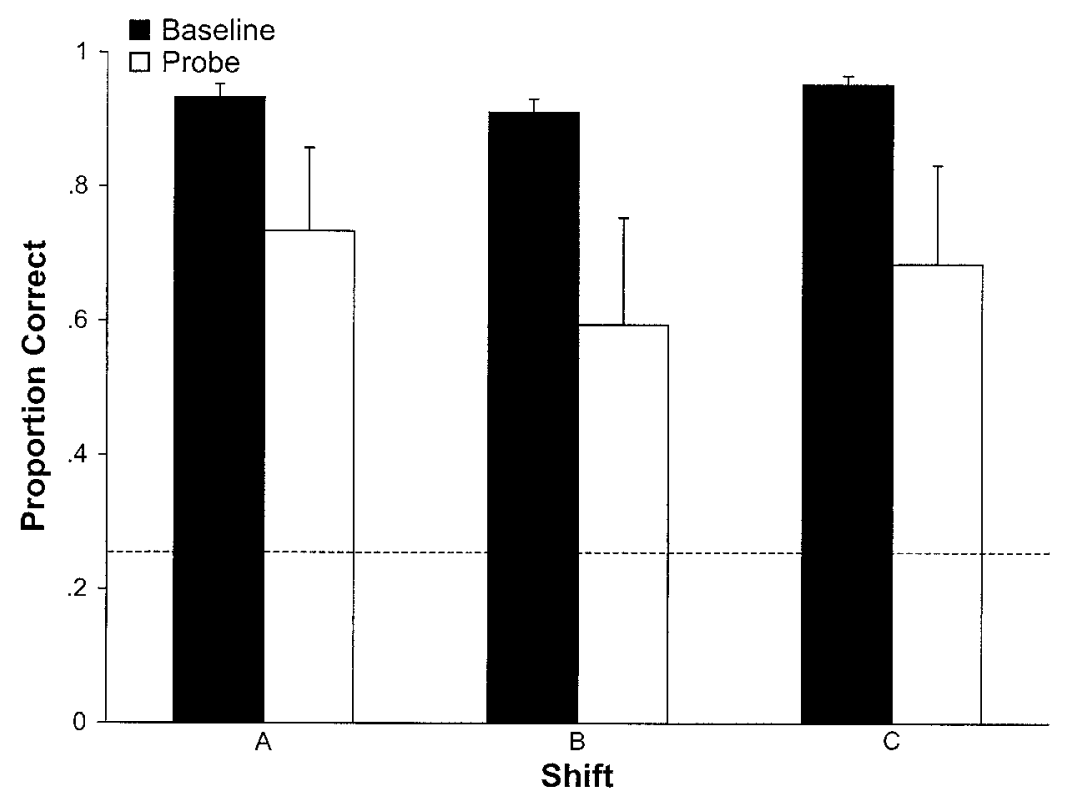

Figure 3. Mean proportion of correct responses during each shift in Experiment 2. The dashed line represents chance performance; error bars represent $S E$.

a light-entrainable or food-entrainableoscillator. If performance is based on an oscillator mechanism, then performance should be high on a test immediately following the elimination of an entrainment stimulus. An oscillator is self-sustaining, meaning that the presentation of stimuli is not required for the continued operation of the oscillator (i.e., it is self-resetting in constant environmental conditions). In contrast, if performance is based on an intervaltiming mechanism (e.g., a pacemaker-accumulator), performance should be low on a test immediately following the elimination of timing stimuli because the omitted stimuli would fail to reset the interval timer. An intermediate result might suggest the operation of multiple timing mechanisms.

\section{Method}

Subjects. The rats from Experiment 1 were also used in Experiment 2.

Apparatus. The apparatus was the same as in Experiment 1.

Procedure. The rats received 15 days of training (described in Experiment 1). The light-cycle transitions were then omitted so that the light remained on throughout the night and the following day prior to the probe test. The meal after Shift $\mathrm{C}$ was also omitted on the day prior to testing. This procedure was carried out in the same manner as in Experiment 1, except that each shift (A, B, and C) was terminated without food when 70 responses occurred at any single location per shift. All responses were recorded, and no reward was given during the probe tests.

\section{Results}

In Figure 3, the proportion of correct responses in each shift during the 15 sessions of training prior to cue omission (baseline) and after cue omission (probe) are plotted. A $2 \times 3$ ANOVA with conditions (baseline vs. probe) and shifts as the repeated measures revealed that there was a significant effect of condition $[F(1,9)=$ 13.41]. There were no significant effects of shift, and no shift $\times$ condition interaction. The mean difference between baseline and probe was $.26(S E=.071)$. Performance during the probe tests was significantly above chance $(.25)$. The same conclusions were reached when the analysis was restricted to the initial $60 \mathrm{sec}$ of data.

\section{Discussion}

The elimination of the posttraining meal and the lightcycle transition did not diminish time-place performance. The rats approached the correct locations on nonrewarded probes at above-chance levels, despite the elimination of putative resetting cues (posttraining meal, light-cycle transition, food pellets during probe shifts); this observation is consistent with a circadian mechanism because such a mechanism involves a self-sustaining oscillator that persists in the absence of resetting stimuli. The cues that were eliminated in the present experiment are the cues for the light-entrainable and feeding-entrainable circadian oscillators. However, performance during probe testing was reliably lower than baseline performance; this observation is consistent with an interval mechanism. Therefore, the rats used both interval and circadian cues. The data do not preclude the possibility that other cues could serve to reset the interval timer. Handling of the rats, the noise level in the building, temperature in the laboratory, and the occurrence of daily shifts could serve as possible cues. In this experiment, we sought to eliminate the most salient cues. One way to test for the importance of the cues listed above is to successively elim- 
inate each cue. If these cues serve to reset an intervaltiming mechanisms, performance would be expected to decline with each successive cue elimination, with performance reaching chance level when all reset cues are eliminated.

\section{GENERAL DISCUSSION}

Rats were trained to search for food at three locations that predictably had food according to the time of day. After training, the rats restricted their visits primarily to the location that was correct for each time of day. When the middle search (B) was tested unusually early or late, the rats did not reliably approach the location that usually provided food in the middle shift. This suggests that (1) the rats were sensitive to the temporal spacing of the shifts, and (2) the temporal order of these events was not sufficient to explain time-place performance. When the posttraining meal and light-cycle transitions in the colony were eliminated, the rats searched at the correct locations. These results suggest that the rats used both interval and circadian cues.

Although the data suggest a role for a self-sustaining circadian-oscillator mechanism, the present data do not indicate whether the circadian oscillator used by the rats was entrained to the light cycle or to the daily feeding schedule. One approach that can be used to decide between these alternatives is to lesion the suprachiasmatic nucleus ( $\mathrm{SCN}$ ), which is known to be the site of the lightentrainable oscillator (Moore \& Eichler, 1972; Stephan $\&$ Zucker, 1972). If the rats can continue to complete the time-place task with lesions to the SCN, this would indicate that the light-entrainable oscillator is not necessary for time-place performance. Although it is well established that the feeding-entrainable circadian oscillator resides outside the $\mathrm{SCN}$, localizing this oscillator has been difficult (see Mistlberger, 1994). One promising recent hypothesis is that the parabrachial nucleus is involved in daily food anticipation because lesions to this region attenuate or eliminate food-anticipatory activity (Davidson, Cappendijk, \& Stephan, 2000).

It is unlikely that the feedings during each shift entrained a feeding-entrainable oscillator, given the available information about the threshold for the feeding-entrainable oscillator. Stephan (1997) estimated that $25.2 \mathrm{kcal}$ is near the threshold for entrainment to a daily meal. The rats in the present study obtained approximately $3.7 \mathrm{kcal}$ in each shift. However, it is possible that smaller estimates of an entrainment threshold could be measured when different responses are appropriate at different times of day, as in the present experiment. In addition, the daily posttesting ration exceeded the threshold for the feedingentrainable oscillator.

A circadian mechanism may be described from two perspectives. According to an entrainment mechanism (see Mistlberger, 1994), entrainment cues (i.e., food) at each time of day function to entrain a circadian oscillator in time-place learning. Therefore, three circadian oscillators would be required for the present task; four os- cillators would be implicated by previous time-place experiments, according to an entrainment mechanism (see Biebach et al., 1991). Because the limits of timeplace learning (i.e., the maximum number of times and places that can be associated) have not been established, the entrainment proposal risks a challenge on the grounds of parsimony. In contrast, a phase-discrimination mechanism (see Gallistel, 1990) requires a single circadian oscillator system. According to this proposal, an oscillator system is entrained to a periodic environmental cue (e.g., daily food ration), and the phases of the circadian system are used as cues to visit each location (e.g., at the times corresponding to Shifts A, B, and C). Therefore, a visit to three locations per day requires the discrimination of three phases of a single oscillator system.

The present results provide information about timing resolution. It is generally accepted that a circadian oscillator is relevant for the discrimination of events that last an hour or more, but not relevant for shorter intervals (Aschoff, 1998; Gibbon, Fairhurst, \& Goldberg, 1997). However, the short interval in the present time-place task was $0.55 \mathrm{~h}(S E=0.02)$. These data might suggest that the circadian mechanisms apply to intervals considerably shorter than has previously been supposed. Alternatively, the short interval might have been timed by an interval-timing mechanism.

\section{REFERENCES}

Aschoff, J. (1998). Human perception of short and long time intervals: Its correlation with body temperature and the duration of wake time. Journal of Biological Rhythms, 13, 437-442.

Biebach, H., FAlK, H., \& KreBs, J. R. (1991). The effects of constant light and phase shifts on a learned time-place association in garden warblers (Sylvia borin): Hourglass or circadian clock? Journal of Biological Rhythms, 6, 353-365.

Biebach, H., Gordijn, M., \& Krebs, J. R. (1989). Time and place learning by garden warblers, Sylvia borin. Animal Behavior, 37, 353360.

Brannon, E. M., Wusthoff, C. J., Gallistel, C. R., \& Gibbon, J. (2001). Numerical subtraction in the pigeon: Evidence for a linear subjective number scale. Psychological Science, 12, 238-243.

CARr, J. A., TAN, A. O., \& Wilkie, D. M. (1999). Further evidence that rats use ordinal timing in a daily time-place learning task. Behavioural Processes, 48, 35-48.

CARr, J. A., \& Wilkie, D. M. (1997a). Ordinal, phase and interval timing. In C. M. Bradshaw \& E. Szabadi (Eds.), Time and behavior: Psychological and neurobehavioral analyses (pp. 265-327). New York: Elsevier.

CARr, J. A., \& WiLkiE, D. M. (1997b). Rats use an ordinal timer in a daily time-place learning task. Journal of Experimental Psychology: Animal Behavior Processes, 23, 232-247.

Church, R. M., \& Lacourse, D. M. (1998). Serial pattern learning of temporal intervals. Animal Learning \& Behavior, 26, 272-289.

Davidson, A. J., CappendiJK, S. L. T., \& STEPhan, F. K. (2000). Feedingentrained circadian rhythms are attenuated by lesions of the parabrachial region in rats. American Journal of Physiology: Regulatory, Integrative \& Comparative Physiology, 278, 1296-1304.

Fountain, S. B., Benson, A. M., \& Wallace, D. G. (2000). Number, but not rhythmicity, of temporal cues determines phrasing effects in rat serial-pattern learning. Learning \& Motivation, 31, 301-322.

Gallistel, C. R. (1990). The organization of learning. Cambridge, MA: MIT Press.

GibBon, J. (1991). Origins of scalar timing. Learning \& Motivation, 22, $3-38$. 
Gibbon, J., \& Church, R. M. (1981). Time left: Linear versus logarithmic subjective time. Journal of Experimental Psychology: Animal Behavior Processes, 7, 87-107.

GibBon, J., FAirhurst, S., \& GoldBerg, B. (1997). Cooperation, conflict, and compromise between circadian and interval clocks. In C. M. Bradshaw \& E. Szabadi (Eds.), Time and behavior: Psychological and neurobehavioral analyses (pp. 329-384). New York: Elsevier.

Killeen,P. R, \& Fetterman, J. G. (1988). A behavioral theory of timing. Psychological Review, 95, 274-295.

Krebs, J. R, \& Biebach, H. (1989). Time-place learning by garden warblers (Sylvia borin): Route or map? Ethology, 83, 248-256.

Mist Lberger, R. E. (1994). Circadian food-anticipatory activity: Formal models and physiological mechanisms. Neuroscience \& Biobehavioral Reviews, 18, 171-195.

Mistlberger, R. E., De Groot, M. H. M., Bossert, J. M., \& Marchant, E. G. (1996). Discrimination of circadian phase in intact and suprachiasmatic nuclei-ablated rats. Brain Research, 739, 12-18.

MoORE, R. Y., \& EICHLER, V. B. (1972). Loss of a circadian adrenal corticosterone rhythm following suprachiasmatic lesions in the rat. Brain Research, 42, 201-206.

SAKSIDA, L. M., \& WILKIE, D. M. (1994). Time-of-day discrimination by pigeons, Columba livia. Animal Learning \& Behavior, 22, 143-154.

StAdDon, J. E. R., \& Higa, J. J. (1999). Time and memory: Towards a pacemaker-free theory of interval timing. Journal of the Experimental Analysis of Behavior, 71, 215-251.

STEPHAN, F. K. (1997). Calories affect zeitgeber properties of the feedingentrained circadian oscillator. Physiology \& Behavior, 62, 995-1002.

Stephan, F. K., \& Zucker, I. (1972). Circadian rhythms in drinking and locomotor activity of rats are eliminated by hypothalamic lesions. Proceedings of the National Academy of Sciences, 69, 1583 1586.

Stevens, S. S. (1951). Mathematics, measurement, and psychophysics. In S. S. Stevens (Ed.), Handbook of experimental psychology (pp. 149). New York: Wiley.

WeARdEn, J. H. (2002). Traveling in time: A time-left analogue for humans. Journal of Experimental Psychology: Animal Behavior Processes, 28, 200-208.

Widman, D. R., Gordon, D., \& Timberlake,W. (2000). Response cost and time-place discrimination by rats in maze tasks. Animal Learning \& Behavior, 28, 298-309.

Wilkie, D. M., \& Willson, R. J. (1992). Time-place learning by pigeons, Columba livia. Journal of the Experimental Analysis of Behavior, 57, 145-158.

(Manuscript received February 28, 2002; revision accepted for publication June 11, 2002.) 